\title{
Unit Commitment in Power System by Combination of Dynamic Programming, Genetic Algorithm and Particle Swarm Optimization
}

\author{
Fazlollah Rouhi ${ }^{1}$ and Reza Effatnejad ${ }^{2 *}$ \\ 'Department of Electrical Engineering, Alborz Branch-University of Science and Research, \\ Islamic Azad University-Alborz-Iran; fazlolaruhi@yahoo.com \\ 2Electrical Engineering Department, Karaj Branch, Islamic Azad University, Alborz, Iran; Reza.efatnejad@kiau.ac.ir
}

\begin{abstract}
In this paper, a hybrid method is proposed for dynamic programming, genetic algorithm and particle swarm optimization in order to solve the unit commitment of power systems considering system security constraints. The condition of the units to be on or off and economic dispatch are determined and solved by considering system security constraints through previously mentioned combination method.

The simulation result is shown that hybrid system is a good methodology for unit commitment. The result is evaluated for experimental case study considering all constraint in power system; the results obtained are acceptable.
\end{abstract}

Keywords: Combinatorial Solution Strategy, Dynamic Programming (DP), Genetic Algorithm (GA), SCUC Security Constrained Unit Commitment, Particle Swarm Optimization (PSO)

\section{Introduction}

Unit commitment (UC) in power system includes determination of planning the units to be on and off in order to supply the forecasted load in a period of time. Constraints such as load balance, system spinal reserve, unit generation limit, pollution etc. are included in this planning schedule can minimize the cost of power system operation.

There are two processes ahead in solving the unit commitment problem. First to determine the units to be on or off. The numbers " 1 " and " 0 " represent the condition of the units whether is on or off respectively. In the other process the economic dispatch should be analyzed, in the other words, how much power should be allocated to each unit from whole generated power over a period of time when the unit is on.

But solving the UC problem alone will not solve the problem, since the network security constraints are not considered. Generation configuration is considered in power system and security constraints are determined for UC operation function. Security constrained unit commitment (SCUC) is evaluated for example limitation of transmission line ${ }^{1}$.

The goal of SCUC is generation configuration planning with the lowest cost and reliability².

Large modern units are equipped with multi-valves turbines and the unit output would have a waveform curve which is called valve point effect ${ }^{3}$. By considering this form of output the problem will be so complicated.

The complexity of this problem makes us to get through dimensions and limitations of this problem and considering proposed a new method for optimization.

Thus, efforts are to be made to supply the load demand of costumers in an economic and secure way.

In this paper we attempt to present a suitable and economic method to determine the unit commitment

*Author for correspondence 
arrangement considering system security constraints which may lead to minimizing the cost and improving the security level of the network.

\section{Proposed Algorithm to Solve the SCUC Problem}

In order to solve the SCUC problem using the combination of binary PSO algorithm and genetic algorithm which are included with dynamic programming (DP $)^{1,4}$. First the condition of the units to be on or off should be determined one by one and then each unit generation will be defined. Sometimes the PSO optimization algorithm involves local optimization and would not be able to define the global optimization, by using the mutation and cutting operators of genetic algorithm it would be possible to get close to local optimization. Using these operators the convergence of data exchange is done better and response is searched to find the global optimization. Therefore, these operators improve the functionality of PSO algorithm in getting away from local optimal point ${ }^{5}$.

In PSO algorithm, if all the group members considered as neighboring of a single member then the best member of the group will be always the leader of the other members.

Referring to the algorithm motion law and by considering the equation $\mathrm{p}_{\text {best }}=\mathrm{g}_{\text {best }}=\mathrm{x}_{\mathrm{i}}$ for the best member of the group, it can be understand that $\mathrm{p}_{\text {best }}=$ $\mathrm{g}_{\text {best }}$ has no leader and is moved based only on its velocity vector.

The algorithm is shown that suitable result.

In this section, the operation of PSO algorithm is improved using genetic algorithm operators "crossover" and "mutation". In the proposed method the crossover operator is used to transfer data between two optimal particles; and mutation operator is used to escape from the local optimum. For every iteration by choosing two particles as parents randomly and using the crossover operator, a new member is added to set. By using the mutation operator, fraction of particles is thrown to random locations in order to replace the pervious "gbest" in case of finding better points. Considering the fact that particles do have memory, the point in using mutation and crossover operators in proposed algorithm, is that, the convergence velocity of PSO algorithm will remain at its pervious value and if by using these operators a more optimum location is found, the particle will move to that location, otherwise the movement won't be deviated. Therefore the convergence velocity will remain standstill. It is possible to use equation below to apply mutation operator on particles ${ }^{6,7}$.

$$
\begin{aligned}
\Delta \mathrm{X}= & \left(\operatorname{var}_{\mathrm{hi}}-\mathrm{X}_{\mathrm{i}}\right) *\left(1-\operatorname{rand}^{(1-(\text { iter/maxiter }) 5}\right) \\
& -\left(\mathrm{X}_{\mathrm{i}}-\operatorname{var}_{\mathrm{lo}}\right) *\left(1-\operatorname{rand}^{(1-(\text { iter/maxiter }) 5)}\right.
\end{aligned}
$$

Where:

$\Delta \mathrm{X}$ : Change in the position of members which are mutated.

$\operatorname{var}_{\mathrm{h}}$ : Maximum value of $\mathrm{X}$

$\operatorname{var}_{10}$ : Minimum value of $\mathrm{X}$

rand: Random number generation function which produce numbers between 0 and 1 with monotonous probability distribution.

iter: Loop Counter of algorithm.

maxiter: Maximum number of algorithm loop repeat.

The created mutation using the above equation causes some members to be out of the specified range of search. So the locations which are out of the range of search space are investigated randomly. It should be noted that the memory of PSO algorithm causes the particle swarm movement not to deviated in the case of the locations which are obtained from mutation are not optimum.

So by using mutation operator, convergence velocity won't decrease.

\section{The proposed algorithm is as follows:}

1 - Form the initial population.

2 - Evaluation of each particle current position.

3 - Determine the best location ever experienced by any particle.

4 - Determine the best position experienced by the group of particles up to now.

5 - Set the velocity and new location of each particle.

6 - Apply crossover operators to the particles and adding a new member.

7 - Apply mutation operator to the percentage of particles randomly.

8 - Form a new population.

9 - Check the stopping criterion for the new population, go to step 10 if the criterion is met, go to step 2 if it is failed to fulfill the criteria.

10 - Show new population. 


\section{Applying the Proposed Algorithm to Solve the Problem" SCUC"}

This section is to implement and apply the proposed algorithm. An IEEE 30 buses system, in which two hydroelectric units are added to its buses number 15 and 20, is considered and will have the following steps ${ }^{8,9}$ :

\subsection{First Step}

In this step the initial population is set. Since the power system is consists of $\mathrm{N}$ generation units and also the time period which is considered in this study is a season which every month of it is divided into sections. And for each section, three levels of load are considered includes minimum, average and maximum. 18 time intervals are considered in planning so every group of population can be shown using a $\mathrm{N} * 18$ matrixes which is specified through following equation:

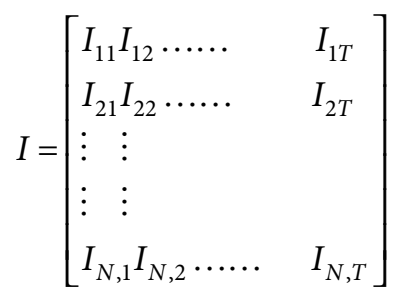

Where " $\mathrm{T}$ " represents the time intervals in the schedule and here is equal to 18. Also "Ix, $y$ " is the position of the " $x$ " unit in the time period of " $y$ ". It should be noted that each element of matrix "I" is determined randomly and is equal to 1 or 0 and the previously mentioned matrix is only related to one group of population and in order to produce the whole population, several matrix should be created like matrix "I" equal to number of existing groups in population.

\subsection{Second Step}

After the initial population is generated spinning reserve constraints and periodic inspections and overhauls are checked and if any of these two constraints are not meet various methods can be used to satisfy these constraints. In this study, a method based on the "priority list" of the units is used to satisfy these constraints.

$$
\mathrm{T}_{\mathrm{i}, \text { on }}^{\mathrm{t}+1}=\left\{\begin{array}{ll}
\mathrm{T}_{\mathrm{i}, \text { on }}^{\mathrm{t}}+1 & \mathrm{I}=1 \\
\mathrm{~T}_{\mathrm{i}, \text { on }}^{\mathrm{t}} & \mathrm{I}=0
\end{array}\right\}
$$

Where " $\mathrm{I}=1$ " represents the unit is turned on and "I $=0$ " means it's off and these values are obtained from "I" matrix and " $T_{i, o n}^{t}$ " is duration of time in which the unit is turned on until the time period " $T$ " of planning schedule. Also, it is possible to define an initial activity duration for each unit which means how many times each unit have been on at the beginning of planning schedule and the duration of this condition can be shown by a positive number and the off condition is shown by a negative number.

\subsection{Third Step}

After determining the initial population and satisfying the mentioned constraints listed in the previous step for each of the categories, now, the production costs should be determined for each category of the units status (on or off). To determine the production cost the "economic dispatch" should be solved considering the units which are on and in order to do so the following steps should be taken into account:

1 - Generate the initial population.

2 - Supply power generation limit.

3 - Supplying "supply and demand balance" constraints.

4 - Supplying maximum allowable pollution constraints.

5 - Supplying generation limit in hydropower units.

6 - Supplying transmission lines limit constraint.

7 - Determine the value of each group, optimal level of personal and global (pbest and gbest).

8 - Set the stop criterion for the economic dispatch algorithm.

There are various ways to determine the stop time of the algorithm. But one of the most common ways is considering a certain number of iterations. In this paper 1000 iterations is considered.

\subsection{Fourth Step}

After determining the generation cost for each of the categories, in this section, the best answer should be determined so it can be used for next steps. Therefore, the 
Table 1. Proposed load pattern for test system

\begin{tabular}{|c|c|c|c|c|c|c|c|c|c|}
\hline \multicolumn{3}{|c|}{ load Level } & \multirow{2}{*}{ Section } & \multirow{2}{*}{ mount } & \multicolumn{3}{|c|}{ load Level } & \multirow{2}{*}{ Section } & \multirow{2}{*}{ mount } \\
\hline maximum & Average & minimum & & & maximum & Average & minimum & & \\
\hline 283.14 & 198.2 & 133.81 & 1 & \multirow[b]{2}{*}{7} & 242.88 & 170.02 & 111.72 & 1 & \multirow[b]{2}{*}{1} \\
\hline 277.48 & 194.24 & 131.14 & 2 & & 240.55 & 168.39 & 110.655 & 2 & \\
\hline 246.77 & 172.74 & 126.08 & 1 & \multirow[b]{2}{*}{8} & 240.137 & 168.01 & 127.47 & 1 & \multirow[b]{2}{*}{2} \\
\hline 248.66 & 174.06 & 127.04 & 2 & & 243.26 & 170.28 & 129.19 & 2 & \\
\hline 251.47 & 176.03 & 138.31 & 1 & \multirow{2}{*}{9} & 292.26 & 240.58 & 1131.81 & 1 & \multirow[b]{2}{*}{3} \\
\hline 251.1 & 175.76 & 138.10 & 2 & & 351.37 & 245.96 & 158.46 & 2 & \\
\hline 250.01 & 175.01 & 116.71 & 1 & \multirow{2}{*}{10} & 425.15 & 297.61 & 161.56 & 1 & \multirow{2}{*}{4} \\
\hline 249.74 & 174.82 & 116.58 & 2 & & 402.73 & 281.91 & 153.04 & 2 & \\
\hline 249.20 & 174.44 & 140.78 & 1 & \multirow{2}{*}{11} & 389.21 & 272.45 & 146.30 & 1 & \multirow[b]{2}{*}{5} \\
\hline 251.37 & 175.96 & 141.10 & 2 & & 324.34 & 227.04 & 121.92 & 2 & \\
\hline 255.28 & 178.70 & 133.47 & 1 & \multirow{2}{*}{12} & 294.34 & 206.03 & 140.87 & 1 & \multirow[b]{2}{*}{6} \\
\hline 248.39 & 173.87 & 129.86 & 2 & & 275.69 & 192.98 & 9131.95 & 2 & \\
\hline
\end{tabular}

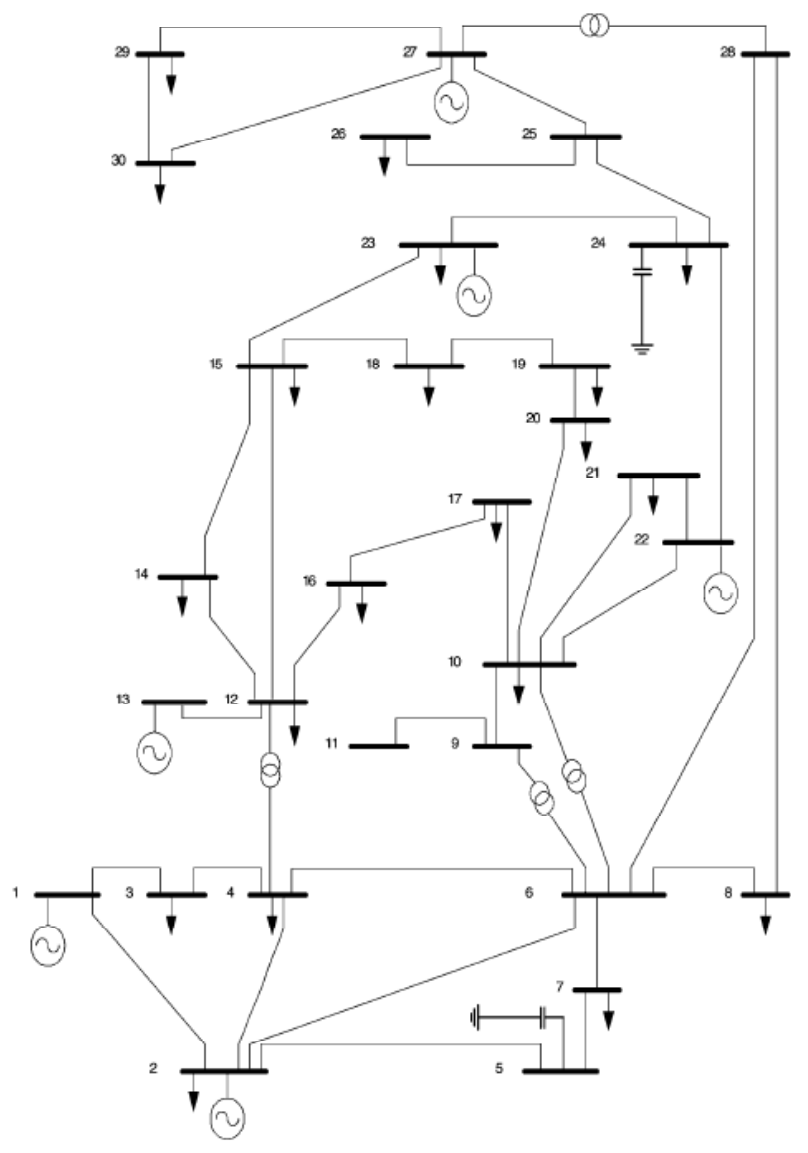

Figure 1. IEEE 30 Buses Test System. best answer for each category and group is determined. It should be noted, definition for best response is to satisfy all the constraints and do have the minimum generation cost.

\subsection{Fifth Step}

New population is determined according to the best obtained from the previous steps and the proposed H-DP-PSO-GA hybrid algorithm.

\subsection{Six Step}

The algorithm introduced in the previous steps is applied on the proposed test system and the result of the simulation will be analyzed.

\section{Determining Load Pattern}

Load pattern for a period of one year is specified in the following table and graph. In this study, each Season is divided to three Months and each month are divided into two parts. And three load levels (minimum, average and maximum) are considered for each part, so, 18 load levels is determined for each season. 


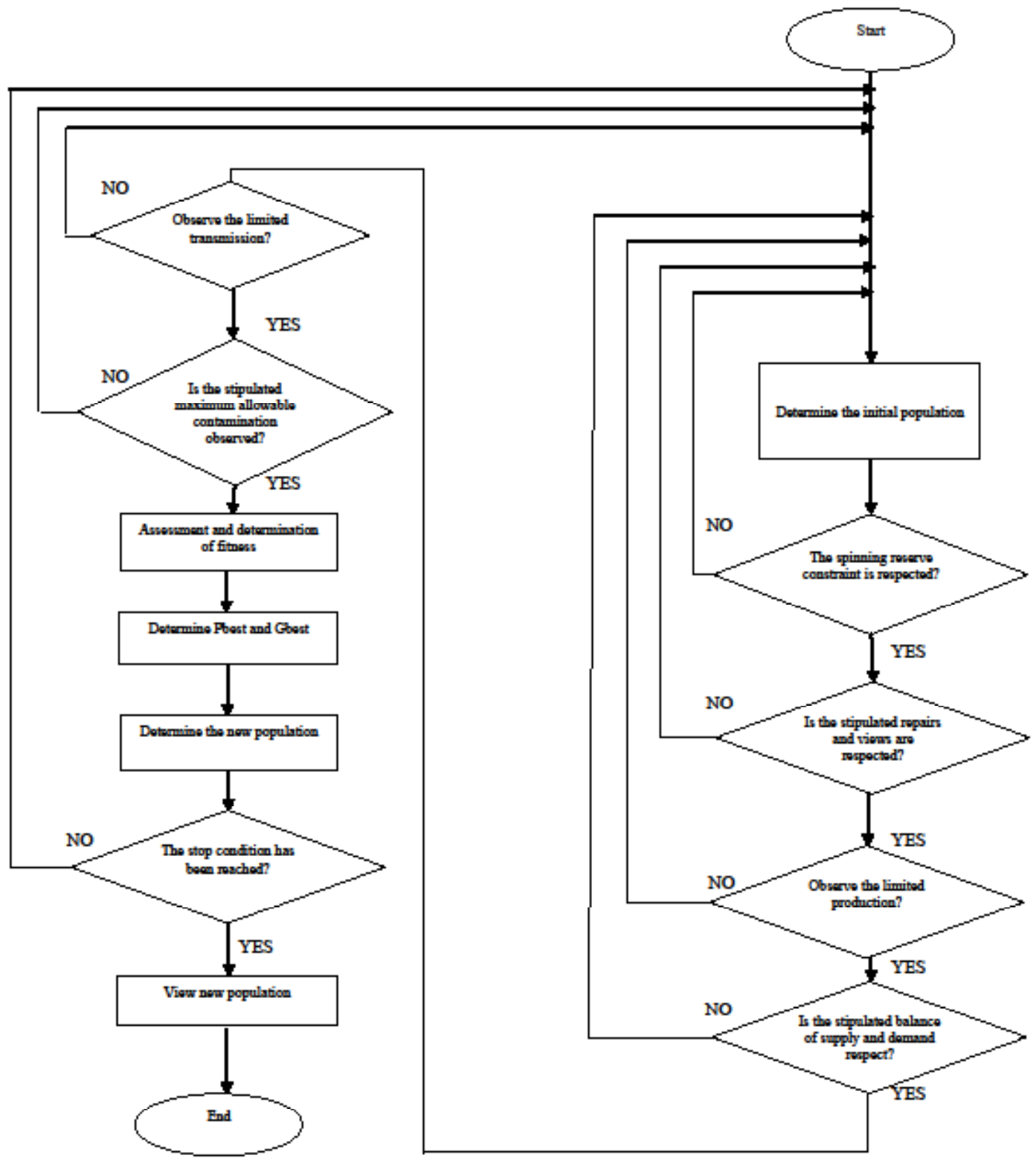

Figure 2. Flowchart of the proposed algorithm.

Since each month is divided into two parts in the proposed test system so, the horizontal axis consists of 24 portions instead of 12 in the figure below.

\section{Simulation and Analysis of Simulation Results}

In order to demonstrate the capabilities of the proposed algorithm, the simulation is carried out as follows:

System contains hydro and thermal units. Thermal units in the system are equipped with the effect of opening the steam valve in order to be close to the real model, also the amount of water in the reservoir of dam is considered as a constraint for the hydroelectric unit, therefore, these units do have limitations to produce electric energy. The spinning reserve constraint is also considered in this scenario. Also, in addition to security constraint, periodic controls and overhauls of the units are considered as other constraints of the system.

Periodic controls and overhauls constraints mean that every single unit is removed from the network after specified period of time to be controlled to see whether 
it needs any overhaul and maintenance process or not. The activity time is considered 18 intervals for hydroelectric units and 15 generators for thermal units. Security constraint put a limit on the power transmission lines in a way that if the power which is carried through the lines exceeded from an acceptable value then the answer will not be accepted. In order to consider these constraints lines 7,12 , and 20 are selected. The maximum permissible power that is considered to cross the lines is 260,100 and $80 \mathrm{MW}$ respectively. Tables 2 and 3 represent the results of implementing the proposed algorithm on the test system. And the total cost is equal to $11,992 \$$. In table 2 , " 0 " and " 1 ", represent whether the unit is turned on or off, respectively.

To illustrate the convergence of the proposed algorithm, the convergence of the algorithm is shown in Figure 4, which shows a proper convergence of the algorithm.

\section{Comparison Between Proposed Method and the PSO Algorithm}

If the PSO algorithm is applied to the system output will be most likely to what is shown in Figure 5. If the proposed algorithm (DP and PSO and GA) is applied the result would be similar to what is shown in figure 4. AS it is obvious the proposed algorithm is more efficient and also the cost is much lower Compare to PSO algorithm alone. By using PSO algorithm the cost is 14581 \$ and if the proposed algorithm is used the cost would be 11992 \$ so it is clear that the proposed algorithm is more capable than PSO algorithm reaching optimal value. There would not any significant change after about 200 iterations by using PSO algorithm but the proposed algorithm reach the cost 12447 \$ after about 200 iterations which is less than the corresponding number of iterations in PSO algorithm and by increasing the number of iterations the proposed algorithm will reach better results values and that is because of the algorithm does not trapped by local optimal which is caused by the mutations of genetic algorithm and that is why the proposed algorithm is more capable.

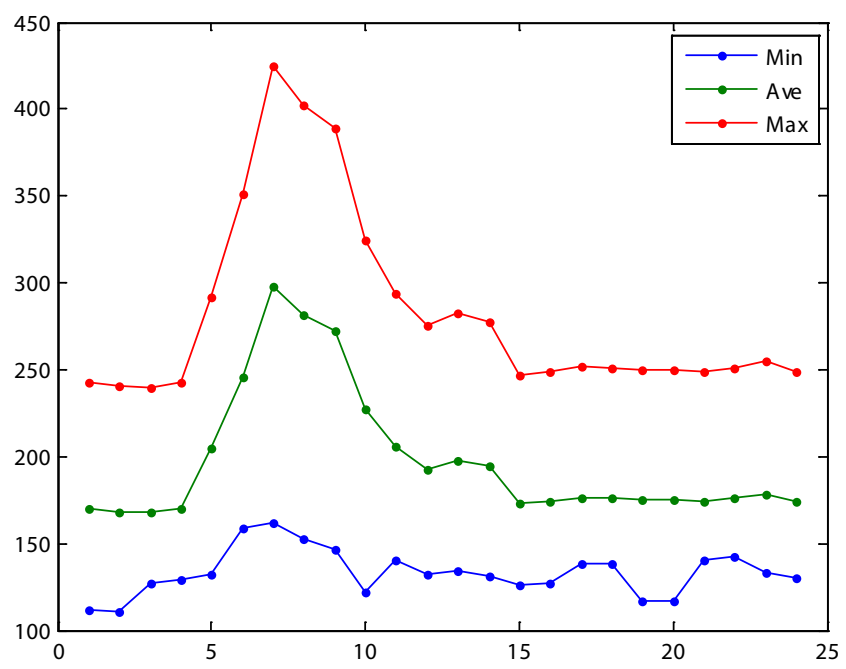

Figure 3. Model of proposed load test system.

Table 2. Results of the simulation

\begin{tabular}{ccccccccccccccccccc}
\hline Period & 1 & 2 & 3 & 4 & 5 & 6 & 7 & 8 & 9 & 10 & 11 & 12 & 13 & 14 & 15 & 16 & 17 & 18 \\
\hline Unit 1 & 1 & 1 & 1 & 1 & 1 & 1 & 1 & 1 & 0 & 1 & 1 & 1 & 1 & 1 & 1 & 1 & 1 & 1 \\
Unit 2 & 0 & 1 & 1 & 1 & 1 & 1 & 1 & 1 & 1 & 1 & 1 & 1 & 0 & 1 & 1 & 1 & 1 & 1 \\
Unit 3 & 1 & 1 & 1 & 1 & 1 & 1 & 1 & 0 & 1 & 1 & 1 & 1 & 1 & 1 & 1 & 1 & 1 & 1 \\
Unit 4 & 0 & 1 & 1 & 1 & 1 & 1 & 1 & 1 & 1 & 1 & 1 & 1 & 0 & 1 & 1 & 1 & 1 & 1 \\
Unit 5 & 0 & 1 & 1 & 1 & 1 & 1 & 1 & 1 & 1 & 1 & 1 & 1 & 1 & 0 & 1 & 1 & 1 & 1 \\
Unit 6 & 1 & 1 & 1 & 1 & 1 & 1 & 1 & 1 & 1 & 0 & 1 & 1 & 1 & 1 & 1 & 1 & 1 & 1 \\
Unit 7 & 1 & 1 & 1 & 1 & 1 & 1 & 1 & 1 & 0 & 1 & 1 & 1 & 1 & 1 & 1 & 1 & 1 & 1 \\
Unit 8 & 1 & 1 & 1 & 1 & 1 & 1 & 1 & 1 & 0 & 1 & 1 & 1 & 1 & 1 & 1 & 1 & 1 & 1 \\
\hline
\end{tabular}


Table 3. The value of each unit of energy produced

\begin{tabular}{lccccccccc}
\hline Period & $\mathbf{1}$ & $\mathbf{2}$ & $\mathbf{3}$ & $\mathbf{4}$ & $\mathbf{5}$ & $\mathbf{6}$ & 7 & $\mathbf{8}$ & $\mathbf{9}$ \\
\hline Unit 1 & 13.5 & 13.5 & 13.5 & 13.5 & 13.5 & 13.5 & 11.5 & 11.5 & 0 \\
Unit 2 & 0 & 13.5 & 13.5 & 13.5 & 13.5 & 13.5 & 13.5 & 13.5 & 13.5 \\
Unit 3 & 5 & 5 & 5 & 5 & 5 & 20.218 & 5 & 0 & 50 \\
Unit 4 & 0 & 35.14 & 16.82 & 5 & 5 & 5 & 5 & 5 & 57.54 \\
Unit 5 & 0 & 49.56 & 5 & 5 & 5 & 98.23 & 5 & 72.7 & 27.42 \\
Unit 6 & 88.21 & 48.30 & 36.20 & 64.58 & 73.13 & 89.30 & 90.77 & 61.74 & 110.7 \\
Unit 7 & 5 & 5 & 100 & 5 & 5 & 5 & 5 & 5 & 0 \\
Unit 8 & 5 & 5 & 60 & 5 & 54.69 & 5 & 5 & 5 & 0 \\
\hline
\end{tabular}

More Table 3. Amount of each unit of energy produced

\begin{tabular}{lccccccccc}
\hline Period & $\mathbf{1 0}$ & $\mathbf{1 1}$ & $\mathbf{1 2}$ & $\mathbf{1 3}$ & $\mathbf{1 4}$ & $\mathbf{1 5}$ & $\mathbf{1 6}$ & $\mathbf{1 7}$ & $\mathbf{1 8}$ \\
\hline Unit 1 & 11.5 & 11.5 & 11.5 & 11.5 & 11.5 & 11.5 & 11.5 & 11.5 & 11.5 \\
Unit 2 & 13.5 & 13.5 & 13.5 & 0 & 11.5 & 11.5 & 11.5 & 11.5 & 11.5 \\
Unit 3 & 5 & 5 & 5 & 5 & 50 & 50 & 5 & 5 & 5 \\
Unit 4 & 60 & 5 & 5 & 0 & 60 & 5 & 60 & 60 & 5 \\
Unit 5 & 1169 & 100 & 100 & 100 & 0 & 100 & 5 & 5 & 100 \\
Unit 6 & 0 & 30.96 & 51.37 & 6.96 & 35.70 & 67.28 & 26.86 & 70.87 & 5039 \\
Unit 7 & 35.31 & 5 & 5 & 5 & 5 & 5 & 5 & 5 & 5 \\
Unit 8 & 5 & 5 & 60 & 5 & 5 & 5 & 5 & 5 & 60 \\
\hline
\end{tabular}

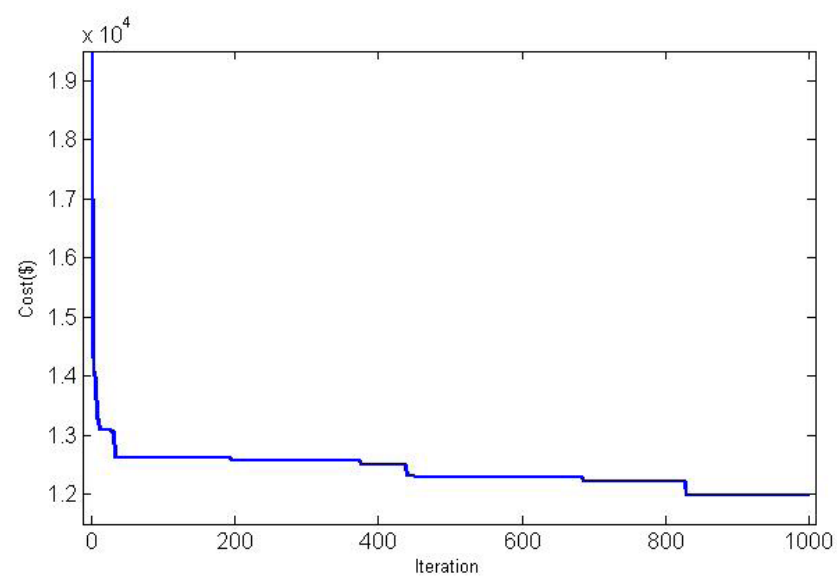

Figure 4. Convergence curves of the proposed Algorithm (H DP PSO GA).

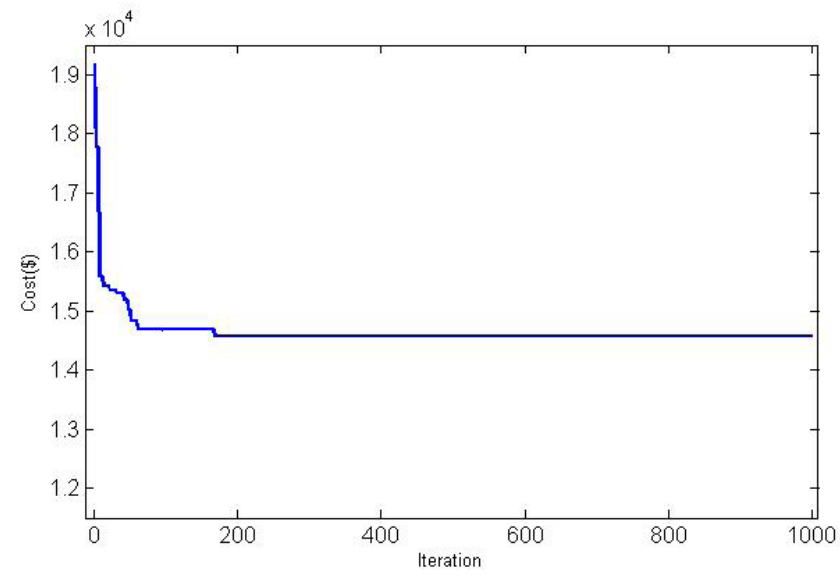

Figure 5. Convergence curve of PSO algorithm. 


\section{Conclusion}

In our proposed method, SCUC problem has both binary variables (on/off condition of the units in form of 1/0) and real variables (real power generation) and the challenge in solving such problems is to establish a logical connection between these two groups of variables. In this research the relationship between these two variable established using PSO and binary algorithms together, then in order to prevent the PSO algorithm from involving in/ trapped in local optimal points combined it with GA algorithm. By considering DP in solving process, repetitive calculation is avoided and Optimal response is achieved. Solving the problem by using proposed algorithm resulted in more optimal responses and supplying costumers economically as it is shown in convergence curves.

\section{References}

1. Ma H, Shahidehpour M. Unit commitment with transmission security and voltage constraints. IEEE Trans Power Syst. 1999; 14(2).

2. Shahidehpour M, Yamin H, Li Z. Market Operations in Electric Power Systems: Forecasting, Scheduling and Risk Management. New York: Wiley; 2002.
3. Fu Y, Shahidehpour M, Li Z. Security-constrained unit commitment with AC constraints. IEEE Trans Power Syst. 2005; 20(2):1001-13.

4. Rong A, Hakonen H, Lahdelma R. A dynamic regrouping based sequential dynamic programming algorithm for unit commitment of combined heat and power systems. Int J Energy Conversion \& Management. 2009; 50:1108-15.

5. Park JB, Lee KS, Shin JR, Lee KY. A particle swarm optimization for economic dispatch with no smooth cost functions. IEEE Trans Power Syst. 2005; 20(1):34-42.

6. Padhy NP. Unit commitment- a bibliographical survey. IEEE Trans Power Syst. 2004; 19(2).

7. Walters DC, Sheble GB. Genetic algorithm solution of economic dispatch with valve point loading. IEEE Trans Power Syst. 1993; 8(3):1325-32.

8. Lin CE, Viviani GL. Hierarchical economic dispatch for piecewise quadratic cost functions. IEEE Trans Power Syst. PAS-103. 1984; 1170-5.

9. Wood AJ, Wolenberg BF. Power Generation, Operation, and Control. 2nd ed. NewYork: Wiley; 1996. p. 131-70. 